PROCEEDINGS OF THE

AMERICAN MATHEMATICAL SOCIETY

Volume 134, Number 1, Pages 23-29

S 0002-9939(05)08273-0

Article electronically published on August 15, 2005

\title{
ON VECTORIAL POLYNOMIALS AND COVERINGS IN CHARACTERISTIC 3
}

\author{
TERESA CRESPO AND ZBIGNIEW HAJTO
}

(Communicated by Jonathan I. Hall)

\begin{abstract}
For $K$ a field containing the finite field $\mathbb{F}_{9}$ we give explicitly the whole family of Galois extensions of $K$ with Galois group $2 S_{4} * Q_{8}$ or $2 S_{4} * D_{8}$ and determine the discriminant of such an extension.
\end{abstract}

\section{INTRODUCTION}

The motivation of this work is the problem of resolution of singularities in positive characteristic, more precisely the ideas presented by S.S. Abhyankar in [4]. Following Abhyankar, loc. cit. Section 18, let $N_{k, t}^{d}$ denote a neighborhood of a simple point on a $d$-dimensional algebraic variety over an algebraically closed field $k$ of characteristic $p$ from which we have deleted a divisor having a $t$-fold normal crossing at the simple point and let $\pi_{A}^{L}\left(N_{k, t}^{d}\right)$ be the set of all Galois groups of finite unramified local Galois coverings of $N_{k, t}^{d}$. In his landmark paper [1], Abhyankar, while working on local uniformization of algebraic varieties in a positive characteristic, proved the inclusion $\pi_{A}^{L}\left(N_{k, t}^{d}\right) \subset P_{t}(p)$, where $P_{t}(p)$ denotes the set of finite groups $G$ such that the quotient $G / p(G)$ of $G$ by the subgroup $p(G)$ generated by its $p$-Sylow subgroups is abelian, generated by $t$ generators. Later, using so-called projective and vectorial polynomials, he proved (see [2, 4]) that $\pi_{A}^{L}\left(N_{k, t}^{d}\right)$ contains $\operatorname{PGL}(m, q)$ and $\operatorname{GL}(m, q)$, for every integer $m>1$ and every power $q>1$ of $p$. Recently D. Harbater et al. [7] proved that for a group $G$ to belong to $\pi_{A}^{L}\left(N_{k, t}^{d}\right)$ it is necessary that $p(G)$ admit an abelian supplement in $G$ of rank $\leq t$. In 4 , Abhyankar exhibited some examples due to G. Stroth of groups contained in $P_{t}(p)$ but not satisfying the abelian supplement condition. In characteristic 3 , and for $t=3$, the Stroth groups are the groups $2 S_{4} * H$, where $2 S_{4}$ denotes a double cover of the symmetric group $S_{4}, H$ is either the quaternion group $Q_{8}$ or the dihedral group $D_{8}$ of order 8 and $*$ denotes central product. In this paper, for $K$ a field containing the finite field $\mathbb{F}_{9}$ of nine elements, we give explicitly the whole family of Galois extensions of $K$ with Galois group $2 S_{4} * H$, and determine the discriminant of such an extension. We note that in [5], the first author provided an explicit construction of $2 S_{4} * Q_{8}$-extensions of fields containing $\mathbb{F}_{9}$ using her previous results on Galois embedding problems based on Serre's trace formula, [9. Here we use a different method of construction combining Abhyankar's embedding criterion [3]

Received by the editors August 4, 2004.

2000 Mathematics Subject Classification. Primary $12 \mathrm{~F} 12$.

This work was partially supported by grant BFM2003-01898, Spanish Ministry of Education. 
and Serre's trace formula, and reach a more explicit and simple formula both for $2 S_{4} * Q_{8^{-}}$and $2 S_{4} * D_{8}$-extensions as well as an explicit formula for the discriminant of such extensions. The explicit determination of the discriminant of these extensions is a step towards local uniformization for three-dimensional varieties in positive characteristic.

\section{Preliminaries}

Let us first recall the definitions and fix the notation. We denote by $2 S_{n}$ one of the two double covers of the symmetric group $S_{n}$ reducing to the nontrivial double cover $2 A_{n}$ of the alternating group $A_{n}$, and by $H$ either the quaternion group $Q_{8}$ or the dihedral group $D_{8}$, double covers of the Klein group $V_{4}$. Let $K$ be a field of characteristic different from 2 and let $\widetilde{L} \mid K$ be a Galois extension with Galois group the group $2 S_{4} * H$. Then if $L$ is the field fixed by the center of $2 S_{4} * H$, we have $\operatorname{Gal}(L \mid K) \simeq S_{4} \times V_{4}$, and for $L_{1}, L_{2}$ the fixed subfields of $L$ by $V_{4}$ and $S_{4}$, respectively, we have $\operatorname{Gal}\left(L_{1} \mid K\right) \simeq S_{4}$ and $\operatorname{Gal}\left(L_{2} \mid K\right) \simeq V_{4}$. Therefore we obtain the whole family of Galois extensions with Galois group $2 S_{4} * H$ of a field $K$ by constructing the whole family of $2 S_{4} * H$-extensions containing a given arbitrary $S_{4}$-extension of the field $K$. Let us now be given a polynomial $f(X) \in K[X]$ of degree 4 with Galois group $S_{4}$ and splitting field $L_{1}$ over $K$. We want to determine when $L_{1}$ is embeddable in a Galois extension of $K$ with Galois group $2 S_{4} * H$. This fact is equivalent to the existence of a Galois extension $L_{2} \mid K$ with Galois group $V_{4}$, disjoint from $L_{1}$, and such that, if $L$ is the compositum of $L_{1}$ and $L_{2}$, the Galois embedding problem

$$
2 S_{4} * H \rightarrow S_{4} \times V_{4} \simeq \operatorname{Gal}(L \mid K)
$$

is solvable. We recall that a solution to this embedding problem is a quadratic extension $\widetilde{L}$ of the field $L$, which is a Galois extension of $K$ with Galois group $2 S_{4} * H$ and such that the restriction epimorphism between the Galois groups $\operatorname{Gal}(\widetilde{L} \mid K) \rightarrow \operatorname{Gal}(L \mid K)$ agrees with the given epimorphism $2 S_{4} * H \rightarrow S_{4} \times V_{4}$. If $\widetilde{L}=L(\sqrt{\gamma})$ is a solution, then the general solution is $L(\sqrt{r \gamma}), r \in K^{*}$. Given a Galois extension $L_{1} \mid K$ with Galois group $S_{4}$, in order to obtain all $2 S_{4} * H$ extensions of $K$ containing $L_{1}$, we have to determine all $V_{4}$-extensions $L_{2}$ of $K$, disjoint from $L_{1}$, and such that the embedding problem (11) is solvable.

Let us consider the double covers $2 S_{4} \rightarrow S_{4}$ and $H \rightarrow V_{4}$ and let $\varepsilon_{1} \in H^{2}\left(S_{4}, \pm 1\right)$, $\varepsilon_{2} \in H^{2}\left(V_{4}, \pm 1\right)$ denote the corresponding cohomology elements. Let $\pi_{1}: S_{4} \times V_{4} \rightarrow$ $S_{4}$ and $\pi_{2}: S_{4} \times V_{4} \rightarrow V_{4}$ be the two projections and let $\pi_{1}^{*}, \pi_{2}^{*}$ be the induced morphisms between the 2-cohomology groups. Then the element $\varepsilon=\pi_{1}^{*}\left(\varepsilon_{1}\right)$. $\pi_{2}^{*}\left(\varepsilon_{2}\right) \in H^{2}\left(S_{4} \times V_{4},\{ \pm 1\}\right)$ corresponds to the double cover $2 S_{4} * H$ of $S_{4} \times V_{4}$. This implies that the element in $H^{2}\left(G_{K},\{ \pm 1\}\right)$ giving the obstruction to the solvability of the embedding problem (11) is equal to the product of the elements giving the obstructions to the solvability of the embedding problems $2 S_{4} \rightarrow S_{4} \simeq \operatorname{Gal}\left(L_{1} \mid K\right)$ and $H \rightarrow V_{4} \simeq \operatorname{Gal}\left(L_{2} \mid K\right)$.

Let us now specify notation by writing $2^{+} S_{n}$ or $2^{-} S_{n}$ depending on whether transpositions in $S_{n}$ lift in the double cover to involutions or to elements of order 4. Let $E=K[X] /(f(X))$, for $f(X)$ the polynomial of degree 4 realizing $L_{1}$, let $Q_{E}$ denote the trace form of the extension $E \mid K$, i.e. $Q_{E}(x)=\operatorname{Tr}_{E \mid K}\left(x^{2}\right)$, and let

$d$ be the discriminant of the polynomial $f(X)$. Let $L_{2}=K(\sqrt{a}, \sqrt{b})$. We denote by $w$ the Hasse-Witt invariant of a quadratic form and by $(\cdot, \cdot)$ a Hilbert symbol. 
By [9] the obstruction to the solvability of the embedding problem $2^{ \pm} S_{4} \rightarrow S_{4} \simeq$ $\operatorname{Gal}\left(L_{1} \mid K\right)$ is equal to $w\left(Q_{E}\right) \cdot( \pm 2, d) \in H^{2}\left(G_{K},\{ \pm 1\}\right)$. By [10], the obstruction to the solvability of $Q_{8} \rightarrow V_{4} \simeq \operatorname{Gal}\left(L_{2} \mid K\right)$ is equal to $(a, b) \cdot(-1, a b) \in H^{2}\left(G_{K},\{ \pm 1\}\right)$ and by e.g. [6], the obstruction to the solvability of $D_{8} \rightarrow V_{4} \simeq \operatorname{Gal}\left(L_{2} \mid K\right)$ is equal to $(a, b) \in H^{2}\left(G_{K},\{ \pm 1\}\right)$ (here we assume that the order 4 elements of $D_{8}$ are mapped on the nontrivial element in $\operatorname{Gal}\left(L_{2} \mid K\right)$ fixing $\left.\sqrt{a b}\right)$.

From now on, we assume that $K$ is a field of characteristic 3 . We write $f(X)=$ $X^{4}+s_{2} X^{2}-s_{3} X+s_{4}$. By computation of the trace form $Q_{E}$, we obtain

$$
w\left(Q_{E}\right)=\left(d s_{2},\left(s_{2}^{2}-s_{4}\right) s_{2}\right) \cdot\left(-1, s_{2}^{2}-s_{4}\right) .
$$

If we further assume that $K$ contains $\mathbb{F}_{9}$, i.e. that $-1 \in K^{2}$, the solvability of the embedding problem (11) is equivalent to

$$
\left(d s_{2},\left(s_{2}^{2}-s_{4}\right) s_{2}\right)=(a, b),
$$

that is, the equality of two Hilbert symbols.

We now recall the isomorphisms $S_{4} \simeq \operatorname{PGL}(2,3)$ and $2^{+} S_{4} \simeq \mathrm{GL}(2,3)$ and state Abhyankar's Embedding Criterion [3] (1.1), and Polynomial Theorem [3] (2.1), (3.7), in our particular case.

Proposition 1. Let $K$ be a field of characteristic 3, and let $M \mid K$ be a Galois extension with Galois group $\mathrm{PGL}(2,3)$. The embedding problem

$$
\mathrm{GL}(2,3) \rightarrow \operatorname{PGL}(2,3) \simeq \operatorname{Gal}(M \mid K)
$$

is solvable $\Leftrightarrow M \mid K$ is the splitting field of a projective polynomial $Y^{4}+c_{3} Y+$ $c_{4} \in K[Y]$. Moreover, if $|K| \geq 9$, the splitting field of the vectorial polynomial $Y\left(Y^{8}+c_{3} Y^{2}+c_{4}\right)$ is a solution to the embedding problem (3).

\section{MAin RESUlts}

Under the hypothesis $\operatorname{char} K=3$, the two equivalent conditions to the solvability of the Galois embedding problem $2^{+} S_{4} \rightarrow S_{4} \simeq \operatorname{Gal}(M \mid K)$ obtained by applying Serre's trace formula and Abhyankar's Embedding Criterion can directly be seen to be equivalent. Indeed, let $M \mid K$ be a Galois extension with the Galois group $S_{4}$ given as the splitting field of a polynomial $f(X)=X^{4}+s_{2} X^{2}-s_{3} X+s_{4} \in K[X]$, let $d$ be the discriminant of $f(X)$, let $x$ be a root of $f(X)$ in $M$ and let $E=K(x)$. Then $M$ is the splitting field of a polynomial of the form $Y^{4}+c_{3} Y+c_{4} \in K[Y]$ if and only if there exists elements $a_{0}, a_{1}, a_{2}, a_{3} \in K$ such that the irreducible polynomial over $K$ of the element $y=a_{0}+a_{1} x+a_{2} x^{2}+a_{3} x^{3}$ has such a form. By computation, this is equivalent to the conditions $a_{0}=-a_{2} s_{2}$ and $Q\left(a_{1}, a_{2}, a_{3}\right):=$ $s_{2} a_{1}^{2}+\left(s_{2}^{2}-s_{4}\right) a_{2}^{2}+s_{2}^{3} a_{3}^{2}+\left(s_{2}^{2}+s_{4}\right) a_{1} a_{3}+2 s_{2} s_{3} a_{2} a_{3}=0$. Now the quadratic trace form $Q_{E}$, for $E=K(x)$, is equivalent to $1+Q$, for $Q$ the quadratic form in $a_{1}, a_{2}, a_{3}$ in the second condition. If we assume $w\left(Q_{E}\right)=(2, d)$, then we have $Q_{E} \sim\langle 1,1,2,2 d\rangle$ (see [9] 3.2) which implies $Q \sim\langle 1,2,2 d\rangle$ and this last quadratic form represents 0 over any field $K$ of characteristic 3 . Reciprocally, assume that $Q$ represents 0 over $K$. Diagonalizing $Q$, we obtain $\left\langle s_{2}, m, s_{2} m d\right\rangle$, with $m=s_{2}^{2}-s_{4}$, and so we have $s_{2} b_{1}^{2}+m b_{2}^{2}+s_{2} m d b_{3}^{2}=0$, for some $b_{1}, b_{2}, b_{3} \in K$, which implies $\left(-d s_{2},-m s_{2}\right)=1$, and so $\left(d s_{2}, m s_{2}\right)=(-1, m d) \cdot(-1,-1)$. Hence we get $w\left(Q_{E}\right)=$ $\left(d s_{2}, m s_{2}\right) \cdot(-1, m)=(-1, d)=(2, d)$.

Theorem 1. Let $K$ be a field of characteristic 3 containing $\mathbb{F}_{9}$, and let $f(X)=$ $X^{4}+s_{2} X^{2}-s_{3} X+s_{4} \in K[X]$, with Galois group $S_{4}$ and $L_{1}$ the splitting field of $f(X)$ 
over $K$. Let $d=s_{4}^{3}+s_{2}^{2} s_{4}^{2}+s_{2}^{4} s_{4}-s_{2}^{3} s_{3}^{2}$ be the discriminant of the polynomial $f(X)$. The family of elements $a, b$ in $K$ such that $(a, b)=\left(d s_{2}, m s_{2}\right)$, where $m=s_{2}^{2}-s_{4}$, can be given in terms of an arbitrary invertible matrix $P=\left(p_{i j}\right)_{1 \leq i, j, \leq 3} \in \mathrm{GL}(3, K)$ as $a=d A, b=s_{2} m F$, where

$$
\begin{aligned}
& A=s_{2} p_{11}^{2}+m p_{21}^{2}+d m s_{2} p_{31}^{2}, \\
& F=d m P_{13}^{2}+d s_{2} P_{23}^{2}+P_{33}^{2}, \quad \text { with } P_{i j}=\left|\begin{array}{cc}
p_{i i} & p_{i j} \\
p_{j i} & p_{j j}
\end{array}\right| .
\end{aligned}
$$

Let $L_{2}=K(\sqrt{a}, \sqrt{b})$ and assume that $L_{2} \mid K$ has Galois group $V_{4}$ and $L_{1} \cap L_{2}=K$ (i.e. that the elements $a, b, a b, d a, d b, d a b$ are not squares in $K)$. Let $L=L_{1} \cdot L_{2}$. For $x$ a root of the polynomial $f(X)$, take $y=a_{0}+a_{1} x+a_{2} x^{2}+a_{3} x^{3}$, with

$$
\begin{aligned}
& a_{0}=-s_{2} a_{2}, \\
& a_{1}=d m \sqrt{-1}\left(n s_{2} p_{11} P_{23}-p_{21} P_{33}+m n p_{21} P_{13}-d s_{2} p_{31} P_{23}\right)+m \sqrt{a}\left(d P_{13}+n P_{33}\right), \\
& a_{2}=d s_{2} \sqrt{-1}\left(p_{11} P_{33}-s_{2}^{2} s_{3} p_{11} P_{23}-m s_{2} s_{3} p_{21} P_{13}-d m p_{31} P_{13}\right)-s_{2} \sqrt{a}\left(s_{2} s_{3} P_{33}+d P_{23}\right), \\
& a_{3}=d m s_{2} \sqrt{-1}\left(s_{2} p_{11} P_{23}+m p_{21} P_{13}\right)+m s_{2} \sqrt{a} P_{33},
\end{aligned}
$$

where $n=s_{2}^{2}+s_{4}$. Then $L(\sqrt{r y}), r \in K^{*}$, is the general solution to the embedding problem

$$
2^{+} S_{4} * D_{8} \rightarrow S_{4} \times V_{4} \simeq \operatorname{Gal}(L \mid K) .
$$

Proof. By [8], 3.2, the equality of Hilbert symbols (2) is equivalent to the $K$ equivalence of quadratic forms

$$
\left\langle d s_{2}, m s_{2}, d m\right\rangle \sim\langle a, b, a b\rangle .
$$

The family of quadratic forms $K$-equivalent to $R:=\left\langle d s_{2}, m s_{2}, d m\right\rangle$ is given by $P^{T} R P$, for $P$ running over $\mathrm{GL}(3, K)$. By diagonalizing $P^{t} R P$, we obtain $\left\langle d A, s_{2} m F, d A s_{2} m F\right\rangle$, with $A$ and $F$ as in the statement. Let $a=d A, b=s_{2} m F$. Now, we have $(a, b)=1 \in H^{2}\left(G_{K(\sqrt{a})},\{ \pm 1\}\right)$ and, as $a \notin K^{2}$ and $L_{1} \cap K(\sqrt{a})=K$, the extension $L_{1}(\sqrt{a}) \mid K(\sqrt{a})$ has Galois group $S_{4}$, and the Galois embedding problem $2^{+} S_{4} \rightarrow S_{4} \simeq \operatorname{Gal}\left(L_{1}(\sqrt{a}) \mid K(\sqrt{a})\right.$ ) is solvable. By the argument preceding Theorem 1, there exist $a_{1}, a_{2}, a_{3} \in K(\sqrt{a})$ such that $Q\left(a_{1}, a_{2}, a_{3}\right)=0$, and for the element $y=a_{0}+a_{1} x+a_{2} x^{2}+a_{3} x^{3}$ we have that $\operatorname{Irr}(y, K(\sqrt{a})$ is a projective polynomial. Also, by Abhyankar's Polynomial Theorem (see Proposition 1), the splitting field of the vectorial polynomial $Y \cdot \operatorname{Irr}(\sqrt{y}, K(\sqrt{a}))$, that is, the field $L_{1}(\sqrt{a})(\sqrt{y})$, is a solution to the Galois embedding problem $2^{+} S_{4} \rightarrow S_{4} \simeq \operatorname{Gal}\left(L_{1}(\sqrt{a}) \mid K(\sqrt{a})\right)$. Now our aim is to compute explicitly such elements $a_{i}$. Diagonalizing $Q$, we obtain $\left\langle s_{2}, m, s_{2} m d\right\rangle$ and from (4) we get that $\left\langle s_{2}, m, s_{2} m d\right\rangle \sim\left\langle A, s_{2} m A F, s_{2} m F d\right\rangle$ and the basis change matrix can be written down explicitly in terms of the matrix $P$. Now the vector $(0, d \sqrt{-1}, \sqrt{a}) \in K(\sqrt{a})^{3}$ anihilates the quadratic form $\left\langle A, s_{2} m A F, s_{2} m F d\right\rangle$, and from it we obtain the values for $a_{1}, a_{2}, a_{3} \in K(\sqrt{a})$ such that $Q\left(a_{1}, a_{2}, a_{3}\right)=0$.

Now we want to see that $L(\sqrt{y}) \mid K$ is a Galois extension with Galois group $2^{+} S_{4} * D_{8}$. By the assumption $L_{1} \cap L_{2}=K$, we have $\operatorname{Gal}\left(L(\sqrt{y}) \mid L_{2}\right) \simeq 2^{+} S_{4}$. We now consider the behaviour of $y$ under the action of $\operatorname{Gal}\left(L_{2} \mid K\right)$. Let $r, s, t$ be the nontrivial elements of $\operatorname{Gal}\left(L_{2} \mid K\right)$ fixing respectively $\sqrt{a b}, \sqrt{b}, \sqrt{a}$. By computation we obtain $y^{s} y=d^{2} h^{2} b$, where $h=m s_{2} p_{31} x^{3}+\left(p_{21}-s_{2}^{2} s_{3} p_{31}\right) x^{2}+\left(m n p_{31}+p_{11}\right) x+$ $s_{2}^{3} s_{3} p_{31}-s_{2} p_{21}$. Now $y \in K(\sqrt{a})(x)$, so $y^{t}=y$ and $y^{r}=y^{s}$, so $L(\sqrt{y})$ is Galois over 
$K$. Now we have $(d h \sqrt{b})^{s}=d h \sqrt{b}$ and $(d h \sqrt{b})^{r}=-d h \sqrt{b}$, so $\operatorname{Gal}\left(L(\sqrt{y}) \mid L_{1}\right) \simeq D_{8}$, with $L(\sqrt{y}) \mid L_{1}(\sqrt{a b})$ cyclic; hence $\operatorname{Gal}(L(\sqrt{y}) \mid K) \simeq 2^{+} S_{4} * D_{8}$.

Remark 1. For the element $y$ given by Theorem 1, we have $\operatorname{Irr}(y, K(\sqrt{a}))=Y^{4}+$ $c_{3} Y+c_{4}$, where

$$
\begin{aligned}
c_{3}= & s_{3} a_{1}^{3}+m a_{1}^{2} a_{2}+s_{2} s_{3} a_{1}^{2} a_{3}+s_{2} s_{3} a_{1} a_{2}^{2}+m s_{2} a_{1} a_{2} a_{3}+m s_{3} a_{1} a_{3}^{2}+s_{3}^{2} a_{2}^{3} \\
& -n s_{3} a_{2}^{2} a_{3}+\left(m^{2}+s_{2} s_{3}^{2}\right) a_{2} a_{3}^{2}+s_{3}^{3} a_{3}^{3}, \\
c_{4}= & \frac{d}{s_{2}^{2}}\left(a_{1} a_{3}-a_{2}^{2}-s_{2} a_{3}^{2}\right)^{2} .
\end{aligned}
$$

Theorem 2. Let the fields $K$ and $L$ and the elements $d, a, b$ and $y$ be as in Theorem 1 , let $\mu=d+(d+1) \sqrt{d}$ and let $\rho=a b+(a b+1) \sqrt{a b}$. Then

1. $L(\sqrt{r \mu y}), r \in K^{*}$, is the general solution to the embedding problem

$$
2^{-} S_{4} * D_{8} \rightarrow S_{4} \times V_{4} \simeq \operatorname{Gal}(L \mid K) .
$$

2. $L(\sqrt{r \rho y}), r \in K^{*}$, is the general solution to the embedding problem

$$
2^{+} S_{4} * Q_{8} \rightarrow S_{4} \times V_{4} \simeq \operatorname{Gal}(L \mid K) .
$$

3. $L(\sqrt{r \mu \rho y}), r \in K^{*}$, is the general solution to the embedding problem

$$
2^{-} S_{4} * Q_{8} \rightarrow S_{4} \times V_{4} \simeq \operatorname{Gal}(L \mid K) .
$$

Proof. For $\sigma \in S_{4} \backslash A_{4}$, we have $\mu^{\sigma} \mu=-(d-1)^{2} d$ and so, $L(\sqrt{\mu y}) \mid K$ Galois. Now $(d-1) \sqrt{-d}$ changes sign under the action of $\sigma$, so $\operatorname{Gal}\left(L(\sqrt{\mu y}) \mid L_{2}\right) \simeq 2^{-} S_{4}$, hence $\operatorname{Gal}(L(\sqrt{\mu y}) \mid K) \simeq 2^{-} S_{4} * D_{8}$.

For $r, s, t \in \operatorname{Gal}\left(L_{2} \mid K\right)$ fixing $\sqrt{a b}, \sqrt{b}, \sqrt{a}$, resp., we have $\rho^{s} \rho=\rho^{t} \rho=$ $-(a b-1)^{2} a b$ and $\rho^{r} \rho=\rho^{2}$, so $L(\sqrt{\rho y}) \mid K$ Galois. Now $(a b-1) \sqrt{-a b}$ changes sign under the action of $s$ and under the action of $t$, so $\operatorname{Gal}\left(L(\sqrt{\rho y}) \mid L_{1}\right) \simeq Q_{8}$, hence $\operatorname{Gal}(L(\sqrt{\rho y}) \mid K) \simeq 2^{+} S_{4} * Q_{8}$.

Combining both arguments, we obtain the third statement in the theorem.

Proposition 2. Let the fields $K$ and $L$ and the elements $s_{2}, s_{3}, s_{4}, d, a, b, m, p_{i j}$ and $y$ be as in Theorem $1 ; \mu, \rho$ as in Theorem 2. We have

$$
\begin{array}{ll}
\operatorname{disc}(L(\sqrt{y}) \mid K) & =d^{104} a^{96} b^{100} D^{2}, \\
\operatorname{disc}(L(\sqrt{\mu y}) \mid K) & =d^{152} a^{96} b^{100} D^{2}(d-1)^{48}, \\
\operatorname{disc}(L(\sqrt{\rho y}) \mid K) & =d^{104} a^{144} b^{148} D^{2}(a b-1)^{48}, \\
\operatorname{disc}(L(\sqrt{\mu \rho y}) \mid K) & =d^{152} a^{144} b^{148} D^{2}(d-1)^{48}(a b-1)^{48},
\end{array}
$$

where

$$
\begin{aligned}
& D=s_{4} p_{11}^{4}-s_{2} s_{3} p_{11}^{3} p_{21}+m s_{2} p_{11}^{2} p_{21}^{2}-m s_{3} p_{11} p_{21}^{3}+\left(m^{2}-s_{2} s_{3}^{2}\right) p_{21}^{4} \\
& +d p_{31}\left(-p_{11}^{3}+m s_{2}^{2} p_{11}^{2} p_{31}-s_{3} p_{21}^{3}+m^{2} s_{2} p_{31} p_{21}^{2}\right)+d^{2} p_{31}^{3}\left(s_{2} s_{3} p_{21}+m p_{11}\right)+d^{3} p_{31}^{4} .
\end{aligned}
$$

Proof. We have $\operatorname{disc}(L(\sqrt{y}) \mid K)=\operatorname{disc}(L \mid K)^{2} \cdot N_{L \mid K}(y)$ and $\operatorname{disc}(L \mid K)=(d a b)^{48}$. Now $N_{L \mid K}(y)=\left(N_{L_{1}(\sqrt{a}) \mid K}(y)\right)^{2}=\left(N_{K(\sqrt{a}) \mid K}\left(c_{4}\right)\right)^{2}$, for $c_{4}$ the degree 0 coefficient in the irreducible polynomial of $y$ over $K(\sqrt{a})$. By computation we obtain $c_{4}=$ $\frac{d}{s_{2}^{2}}\left(a_{1} a_{3}-a_{2}^{2}-s_{2} a_{3}^{2}\right)^{2}$ and, by substituting the values of $a_{1}, a_{2}, a_{3}$ and computing the norm, $N_{K(\sqrt{a}) \mid K}\left(c_{4}\right)=d^{8} b^{4} D^{2}$ for $D$ as in the statement.

To obtain the other three discriminants, it is now enough to compute $N_{L \mid K}(\mu)=$ $N_{K(\sqrt{d}) \mid K}(\mu)^{48}=(d-1)^{48} d^{48}$ and $N_{L \mid K}(\rho)=N_{K(\sqrt{a b}) \mid K}(\rho)^{48}=(a b-1)^{48}(a b)^{48}$. 


\section{EXAmples}

Let $K=k\left(\left(Z_{1}, Z_{2}, Z_{3}\right)\right)$ be the quotient field of the formal power series ring in 3 variables over a field $k$ containing $\mathbb{F}_{9}$. We consider the polynomial

$$
f(X)=X^{4}+Z_{1} X^{2}+Z_{2} X+Z_{3} \in K[X]
$$

i.e. we are taking $s_{2}=Z_{1}, s_{3}=-Z_{2}, s_{4}=Z_{3}$. We can check that the polynomial $f$ has Galois group $S_{4}$ over $K$ and let $L_{1}$ be the splitting field of $f$ over $K$. We consider the extension $L_{2} \mid K$ generated by the elements $\sqrt{d s_{2}}, \sqrt{m s_{2}}, \sqrt{d m}$, which corresponds to taking the matrix $P$ in Theorem 1 to be one of the matrices

$$
P_{1}=\left(\begin{array}{lll}
1 & 0 & 0 \\
0 & 1 & 0 \\
0 & 0 & 1
\end{array}\right) \quad, \quad P_{2}=\left(\begin{array}{lll}
0 & 0 & 1 \\
1 & 0 & 0 \\
0 & 1 & 0
\end{array}\right) \quad, \quad P_{3}=\left(\begin{array}{lll}
0 & 1 & 0 \\
0 & 0 & 1 \\
1 & 0 & 0
\end{array}\right)
$$

We can check that the elements $d s_{2}, m s_{2}, d m, s_{2}, d m s_{2}, m$ are not squares in $K$, and so $L_{2} \mid K$ has Galois group $V_{4}$ and is disjoint with $L_{1} \mid K$. Let $L=L_{1} \cdot L_{2}$. We denote by $y_{i}$ the element $y$ given by Theorem 1 for each of the matrices $P_{i}$, $i=1,2,3$. Then we have

$$
\operatorname{Gal}\left(L\left(\sqrt{y_{i}}\right) \mid K\right) \simeq 2^{+} S_{4} * D_{8}, \quad i=1,2,3,
$$

with $L\left(\sqrt{y_{1}}\right)\left|L_{1}(\sqrt{d m}), L\left(\sqrt{y_{2}}\right)\right| L_{1}\left(\sqrt{m s_{2}}\right), L\left(\sqrt{y_{3}}\right) \mid L_{1}\left(\sqrt{d s_{2}}\right)$ cyclic. The factors appearing in $\operatorname{disc}\left(L\left(\sqrt{y_{1}}\right) \mid K\right)$ are $d, s_{2}, m$ and $s_{4}$, the factors appearing in $\operatorname{disc}\left(L\left(\sqrt{y_{2}}\right) \mid K\right)$ are $d, s_{2}, m$ and $m^{2}-s_{2} s_{3}^{2}$, and the factors appearing in $\operatorname{disc}\left(L\left(\sqrt{y_{3}}\right) \mid K\right)$ are $d, s_{2}, m$. In particular the discriminantial locus remains unchanged when going from $L$ to $L\left(\sqrt{y_{3}}\right)$.

We observe that the elements $d-1$ and $a b-1$, for each choice of $a, b$ among $d s_{2}, m s_{2}, d m$, are invertible elements in the ring $k\left[\left[Z_{1}, Z_{2}, Z_{3}\right]\right]$, and so the discriminant locus will not change if we realize any other of the groups $2 S_{4} * H$ over the same field $K$ by means of Theorem 2 .

\section{REFERENCES}

[1] S.S. Abhyankar, On the ramification of algebraic functions, Amer. J. Math. 77 (1955), 572592. MR0071851 (17:193c)

[2] S.S. Abhyankar, Local fundamental groups of algebraic varieties, Proc. Amer. Math. Soc. 125 (1997), 1635-1641. MR.1403110(97h:14032)

[3] S.S. Abhyankar, Galois embeddings for linear groups, Trans. Amer. Math. Soc. 352 (2000), 3881-3912. MR 1650057 (2000m:12003)

[4] S.S. Abhyankar, Resolution of singularities and modular Galois theory, Bull. Amer. Math. Soc. 38 (2001), 131-169. MR 1816069 (2002a:14013)

[5] T. Crespo, $2 S_{4} * Q_{8}$-extensions in characteristic 3, Proc. Amer. Math. Soc. 132 (2004), 691-695. MR2019944 (2004j:12003)

[6] A. Fröhlich, Orthogonal representations of Galois groups, Stiefel-Whitney classes and HasseWitt invariants, J. Reine Angew. Math. 360 (1985), 84-123. MR0799658 (87h:11028)

[7] D. Harbater, M. van der Put and R. Guralnick, Valued fields and covers in characteristic $p$, Fields Institute Communications 32 (2002), 175-204. MR1928369 (2003i:12008)

[8] T. Y. Lam, The algebraic theory of quadratic forms, Benjamin-Cummings Publ. Co., Reading, MA, 1973. MR0396410 (53:277) 
[9] J.-P. Serre, L'invariant de Witt de la forme $\operatorname{Tr}\left(x^{2}\right)$, Comment. Math. Helv. 59 (1984), 651676. MR0780081 (86k:11067)

[10] E. Witt, Konstruktion von galoischen Körpern der Charakteristik $p$ zu vorgegebener Gruppe der Ordnung $p^{f}$, J. Reine Angew. Math. 174 (1936), 237-245.

Departament d’Àlgebra i Geometria, Universitat de Barcelona, Gran Via de les Corts Catalanes 585, 08007 Barcelona, Spain

E-mail address: teresa.crespo@ub.edu

Zakead Matematyki, Akademia Rolnicza, Al. Mickiewicza 24/28, 30-059 Kraków, POLAND

E-mail address: rmhajto@cyf-kr.edu.pl

Current address: Instytut Matematyki, Politechnika Krakowska, ul. Warszawska 24, 31-155 Kraków, Poland 